\title{
Storage and Release of Subradiant Excitations in a Dense Atomic Cloud
}

\author{
Giovanni Ferioli, ${ }^{1}$ Antoine Glicenstein, ${ }^{1}$ Loic Henriet, ${ }^{2}$ Igor Ferrier-Barbut $\odot,{ }^{1,{ }^{*}}$ and Antoine Browaeys ${ }^{1}$ \\ ${ }^{1}$ Université Paris-Saclay, Institut d'Optique Graduate School, CNRS, \\ Laboratoire Charles Fabry, 91127, Palaiseau, France \\ ${ }^{2}$ Pasqal, 2 Avenue Augustin Fresnel, 91120 Palaiseau, France
}

(Received 18 December 2020; accepted 3 March 2021; published 10 May 2021)

\begin{abstract}
We report the observation of subradiance in dense ensembles of cold ${ }^{87} \mathrm{Rb}$ atoms operating near Dicke's regime of a large number of atoms in a volume with dimensions smaller than the transition wavelength. We validate that the atom number is the only cooperativity parameter governing subradiance. We probe the dynamics in the many-body regime and support the picture that multiply excited subradiant states are built as a superposition of singly excited states that decay independently. Moreover, we implement an experimental procedure to release the excitation stored in the long-lived modes in a pulse of light. This technique is a first step towards the realization of tailored light storing based on subradiance.
\end{abstract}

DOI: 10.1103/PhysRevX.11.021031

Subject Areas: Atomic and Molecular Physics

\section{INTRODUCTION}

The interaction between a single two-level atom and radiation is well understood: The atomic response is described by a resonance frequency and a decay rate. When considering more than one emitter in a volume with dimensions smaller than the transition wavelength, this response may be altered, as was proposed in the pioneering work of Dicke [1,2]. Indeed, light-induced interactions dramatically modify the behavior of the ensemble, and its response becomes collective. In particular, the decay rate of excitations hosted in the ensemble can be starkly modified. Superradiance, i.e., a decay of the excitation at a rate faster than the single-atom one, has been verified experimentally in atomic systems from ions to dilute clouds of atoms [2-6]. The study of its counterpart, namely, subradiance with a decay rate smaller than the atomic one, has been restricted to a handful of works [7]: Direct observations were reported in a pair of ions at variable distance [3] and in molecular systems [8-10]. Recently, subradiance was also observed in a cold, dilute atomic cloud [11-13]; in Rydberg nonlinear quantum optics [14]; and as a line-narrowing in an ordered 2D layer of atoms [15].

Engineering subradiant states has drawn increasing attention since it might pave the way to several applications. For instance, the possibility of storing an excitation in subradiant modes and of addressing it in real time while the

\footnotetext{
*igor.ferrier-barbut@institutoptique.fr
}

Published by the American Physical Society under the terms of the Creative Commons Attribution 4.0 International license. Further distribution of this work must maintain attribution to the author(s) and the published article's title, journal citation, and DOI. excitation is stored has inspired proposals to use it as a storage medium [16-20]. Second, the narrowing of the line associated with subradiant modes and their subsequent enhanced sensitivity to external fields could be a promising application for metrology [16,21,22]. Recent proposals have also suggested using subradiance as a tool for quantum information processing and quantum optics $[23,24]$.

All of these proposals have been formulated in ordered systems with small interparticle distances, $\bar{r} \lesssim \lambda$, where $\lambda$ is the wavelength of the atomic transition. Motivated by these proposals, we take a first step in this direction by exploring the regime $\bar{r}<\lambda$ but in the disordered case using dense clouds of ${ }^{87} \mathrm{Rb}$ atoms, characterized by a peak density $\rho_{0}$ satisfying $\rho_{0} \lambda^{3} \gg 1\left(\bar{r}=\rho_{0}^{-1 / 3}\right)$. Furthermore, the ensembles we produce have a prolate shape with a typical radial size of about $0.5 \lambda$ and an axial size of about $5 \lambda$. Thus, we closely approach Dicke's regime, where many emitters are trapped in a volume comparable to the wavelength of their transition. This regime introduces several important differences with respect to the case of a dilute extended cloud studied in Refs. [11-13]. First, the ensemble is efficiently coupled only to a single mode, and thus the parameter governing the collective properties should be the atom number $N$ [25]. This case is in contrast to a cloud with a volume much larger than $\lambda^{3}$, where this parameter is the optical depth on resonance, which was experimentally shown to govern collective effects $[4,11]$. Second, since in our clouds $k \bar{r} \sim 1(k=2 \pi / \lambda)$, all the terms of the dipoledipole interaction [26] play a role, as opposed to the dilute regime where only a radiative $1 / r$ term is considered [27].

Here, we observe subradiance in the time domain in a cloud operating near Dicke's regime. First, we validate the characteristic dependence on the atom number. Second, we explore the storage of light in long-lived multiply excited 
states. Varying the intensity of the excitation laser, we characterize these subradiant states containing few to many excitations. Our finding supports the idea that multiply excited subradiant states are built as a superposition of singly excited states in random ensembles, similarly to what was recently predicted in ordered 1D systems [19, 28-30]. Finally, we demonstrate dynamical control of subradiance while excitations are stored by releasing it on demand via the application of a laser. This real-time control of the coupling of an ensemble to the electromagnetic modes while it hosts an excitation offers new possibilities for light storage.

\section{EXPERIMENTAL SETUP}

A detailed description of the experimental setup can be found elsewhere [31,32]. Briefly, as sketched in Fig. 1(a), it is composed of four aspherical lenses with large numerical aperture (0.5) in a maltese-cross configuration [33]. We use the $x$ high-resolution optical axis to create an optical tweezer at a wavelength $\lambda_{\text {trap }}=940 \mathrm{~nm}$, with a tunable waist (range 1.8-2.5 $\mu \mathrm{m}$ ). Exploiting a gray-molasses loading on the D1 line, we trap $\simeq 5000{ }^{87} \mathrm{Rb}$ atoms in the largest tweezer at a temperature of about $650 \mu \mathrm{K}$ in a $4.2 \mathrm{mK}$ trap. In this configuration, the trapping frequencies are $\omega_{r} \simeq 2 \pi \times 81 \mathrm{kHz}$ and $\omega_{z} \simeq 2 \pi \times 7 \mathrm{kHz}$, where $\omega_{r}$ and $\omega_{z}$ represent the radial and the axial directions. The central density of the cloud in these conditions is $\rho_{0} / k^{3}=0.3 \pm$ $0.1(\bar{r} \simeq 0.2 \lambda)$, where $k=2 \pi / \lambda$. The trap can then be compressed either by increasing the power of the trapping beam or reducing its waist [32].

We use the $F=2 \rightarrow F^{\prime}=3$ transition on the $\mathrm{D} 2$ line with wavelength $\lambda \simeq 780 \mathrm{~nm}$, line width $\Gamma_{0} \simeq 2 \pi \times 6 \mathrm{MHz}$, and saturation intensity $I_{\text {sat }}=1.6 \mathrm{~mW} / \mathrm{cm}^{2}$. In order to excite the cloud, we switch off the trap and shine a 150-ns-long pulse of resonant light along the $y-z$ direction of Fig. 1(a). This duration is long enough to reach the steady state during the excitation. After $1 \mu \mathrm{s}$, the atoms are recaptured in the tweezer. This time is short enough $\left(<1 / \omega_{r}\right)$ that the density
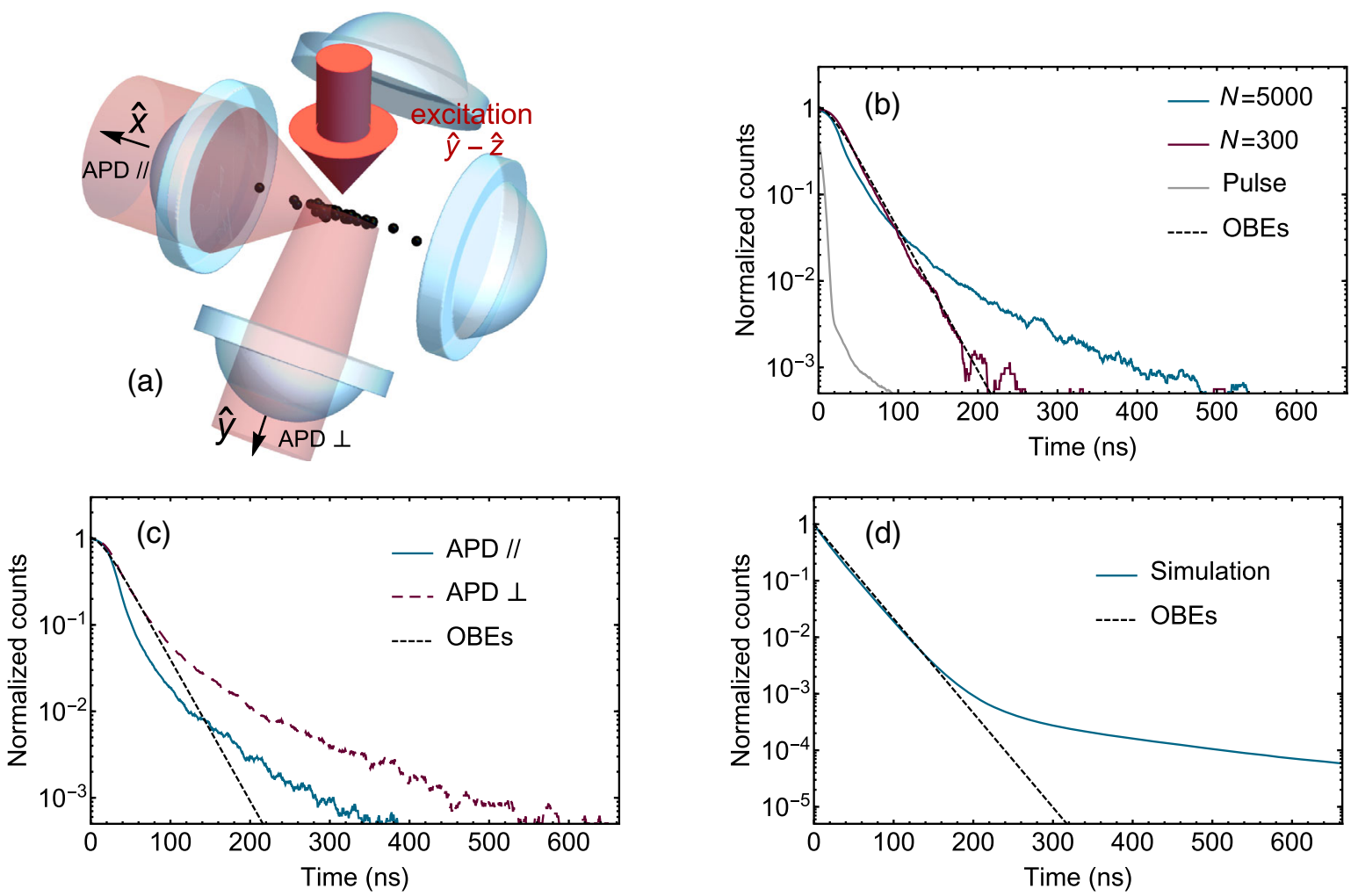

FIG. 1. (a) Schematics of the experimental setup. Four high-numerical-aperture (0.5) lenses collect the fluorescence emitted by the atomic cloud along two axes, which is then fiber-coupled to avalanche photodiodes (APD). The excitation beam is aligned in the vertical direction. The trap beam (not shown) propagates along $x$, which is also the first collection axis. The second collection axis is at $45^{\circ}$ with respect to the excitation direction. (b) Number of photons collected in bins of $0.5 \mathrm{~ns}$ as a function of time after switching off the excitation light (saturation parameter $s \simeq 27$ ) for a cloud containing about 5000 (blue solid line) and 300 atoms (purple solid line), where data are represented with a moving average. These data are obtained by repetition of 20 pulses on 10000 clouds. Gray line: excitation pulse temporal shape. Dashed line: solution of the optical Bloch equations solved for our pulse shape. All curves have been normalized to their steady-state value during the excitation. (c) Time traces collected using the APD aligned along the $y$ direction (APD $\perp$, longdashed purple) and along $x$ (APD//, solid blue). The dashed black line shows the solution of OBEs. (d) Numerical simulations of the experiment using a nonlinear coupled-dipole model $[31,34]$ in an ensemble of 200 atoms with $\rho_{0} / k^{3}=0.3$. Black dashed line: solution of optical Bloch equations for a single atom. 
remains constant during this trap-free time. We repeat the same sequence up to 20 times on the same cloud (depending on the trap geometry), checking that the atom number is reduced by less than $10 \%$ at the end of the pulse sequence. We then repeat this sequence on 3000 to 10000 clouds at a $2-\mathrm{Hz}$ rate to obtain one trace (photon count per 0.5-ns bin versus time). For the largest atom number, $N \simeq 5000$, we typically collect, in the steady state, 0.01 photon per pulse in a 0.5 -ns time bin. The excitation pulse is controlled by means of two acousto-optical modulators in series, ensuring a high extinction ratio. The characteristic rise and fall time is $10 \mathrm{~ns}$; the temporal shape of the pulse is shown in Fig. 1(b) (gray line). The excitation beam waist that is approximately $1 \mathrm{~mm}$ is much larger than the cloud size, so all the atoms experience the same Rabi frequency. We can vary the saturation parameters $s=I / I_{\text {sat }}$ up to $s \simeq 250$, with $I_{\text {sat }}$ the saturation intensity (we have calibrated this intensity independently using dilute clouds). Exploiting the two high-resolution optical axes, we collect the fluorescence light into two fiber-coupled APDs operating in single-photon-counting mode - one aligned along the axial direction of the cloud $(\mathrm{APD} / /)$, the other perpendicularly to it $(\mathrm{APD} \perp)$ - as sketched in Fig. 1(a).

\section{SUBRADIANCE NEAR DICKE'S REGIME}

In Fig. 1(b), we report two time-resolved fluorescence traces recorded along $x$, obtained for two clouds with, respectively, 300 atoms $\left(\rho_{0} / k^{3} \simeq 0.02\right.$, purple line) and 5000 atoms $\left(\rho_{0} / k^{3} \simeq 0.3\right.$, blue line $)$ after the excitation laser has been switched off for $s \simeq 27$. We also show the solution of the optical Bloch equations (OBEs) for a single atom, solved for the measured pulse shape. In the lowatom-number case, the decay of the excitation is very well described by the OBEs, indicating that the atoms act as independent atoms. On the contrary, for a large atom number, the fluorescence decays nonexponentially: First, we observe a decay at a rate larger than the single-atom decay (superradiance), followed by a slower one (subradiance). Moreover, as shown in Fig. 1(c), we observe that superradiance occurs mainly in the axial direction of the cloud, while the emission of subradiant excitation is observed in both directions. The finite switch-off time of the driving pulse limits the superradiant decay that can be observed. For this reason, in the rest of the paper, we focus our attention on the subradiant tail, leaving a detailed study of superradiance for future works.

All the measurements reported here have been performed with resonant and linearly polarized light, in the absence of Zeeman optical pumping, thus exciting a multilevel system. We find that the polarization direction does not impact the observed subradiance. Furthermore, we have observed that subradiance is essentially unchanged within our dynamical range in the presence of a magnetic field and a circularly polarized pulse with prior optical pumping (see the Appendix E), which suggests that the internal structure does not play a major role for subradiance in our regime. Finally, we have checked that subradiance is unchanged when we vary the detuning of the excitation laser around the atomic resonance (Appendix B). This result indicates that radiation trapping of light in the cloud, which is seen as a random walk of the photons before escaping, cannot explain the observed slow decay [35]. For our small, dense cloud - and contrarily to the case of dilute, optically thick clouds [11] - this result is expected, as the photon meanfree path $l_{\mathrm{sc}}=1 /\left(\rho \sigma_{\mathrm{sc}}\right)$ (with $\rho$ the atomic density and $\sigma_{\mathrm{sc}}=3 \lambda^{2} / 2 \pi$ the resonant cross section) is smaller than the mean interparticle distance.

To further support our observations of subradiance, we perform numerical simulations in a simplified setting of two-level atoms. For the large number of atoms involved in the experiment, the $a b$ initio simulation using a master equation is beyond reach, and we therefore resort to approximations. We use a nonlinear coupled-dipoles model $[31,34]$ consisting of a coupled system of OBEs, given in Appendix A. It formally amounts to a mean-field approximation, assuming that the density matrix of the system can be factorized [34,36,37]. Thus, we can take into account saturation effects of individual atoms. We numerically solve the equations for $N=200$ atoms at a density $\rho_{0} / k^{3}=$ 0.3 [Fig. 1(d)]. The results do not feature superradiance, but they do yield subradiance. The origin of the superradiance observed in the experiment and not present in the meanfield simulation is left for future investigations. The prediction of subradiance in our simulations could suggest that the mean-field model is enough to account for our observations. However, as we will show below, it fails to reproduce our results in the strongly saturated regime, even qualitatively. In the remainder of this section, we characterize the observed subradiance as a function of atom number.

To reveal the characteristic scaling of subradiance in our regime, we investigate the crossover between the low-atomnumber regime, where the system behaves as an ensemble of noninteracting emitters, and the large-atom-number one. To adjust the atom number $N$, we release the atoms from the trap and recapture them after a variable time prior to sending the burst of excitation pulses. This technique allows us to reduce the atom number by a factor of more than 10 with negligible heating, thus leaving the cloud sizes unchanged. To analyze the experimental data, we fit the decay with a phenomenological model using either a single exponential decay or the sum of two decays with different characteristic times. The fitting function is decided based on a $\chi^{2}$ criterion (see Appendix $\mathrm{C}$ for more details). The decay time is extracted from data averaged over tens of thousands of realizations and thus corresponds to an average subradiant behavior.

In Fig. 2(a), we report the results of this analysis. We observe that as $N$ grows, a clear subradiant tail appears, and that the characteristic decay time is an increasing function of $N$. To determine the parameter that governs the 

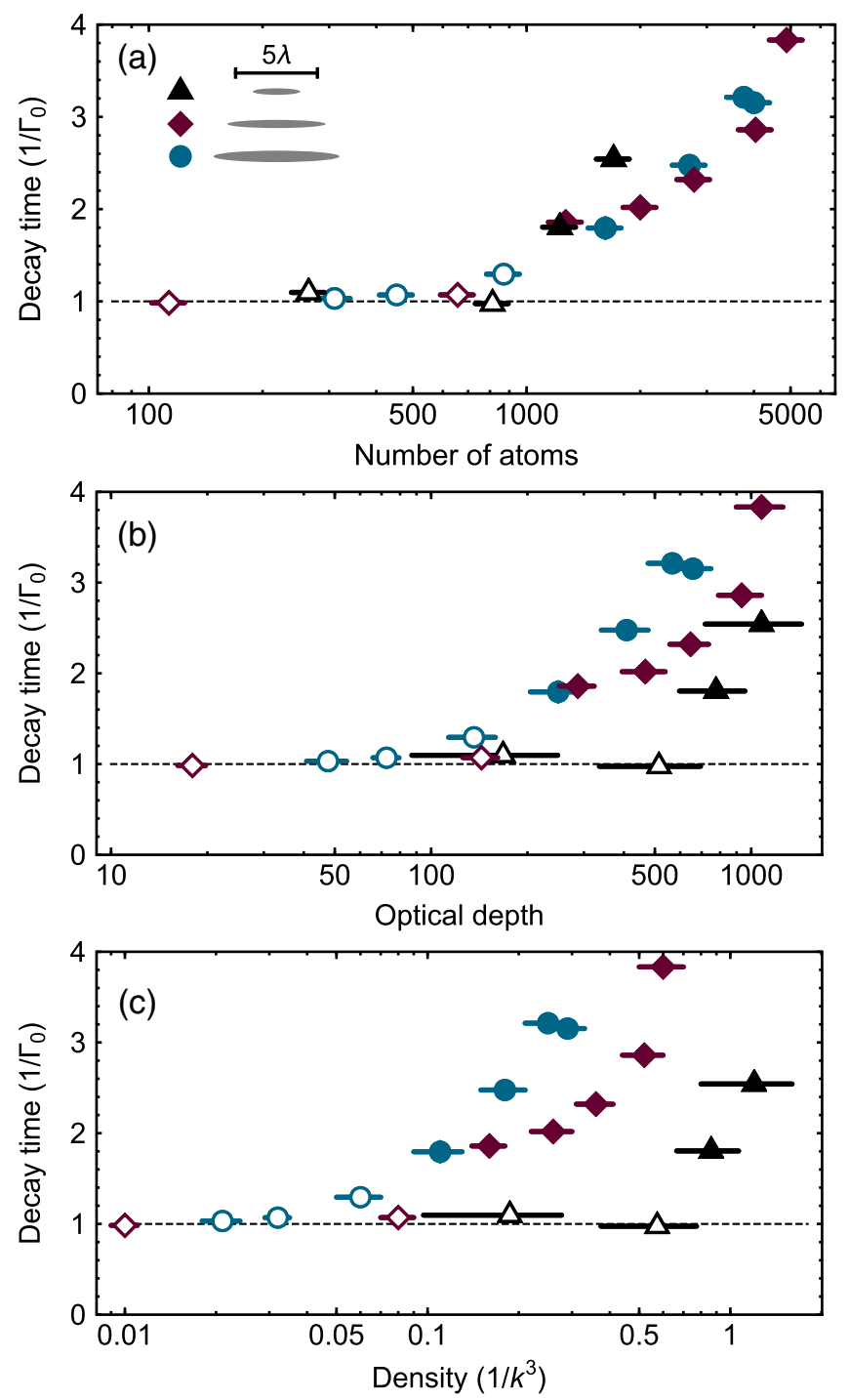

FIG. 2. Decay times in units of the single-atom lifetime $\Gamma_{0}^{-1}$ evaluated by fitting the traces with a sum of two exponential decays (filled symbols) or with a single one (empty symbols), as explained in the text. The three different data sets are obtained in three different geometries, giving cloud Gaussian sizes $\left(l_{r}, l_{x}\right)$ : $(0.7 \lambda, 7.7 \lambda)$ (circles), $(0.5 \lambda, 6.0 \lambda)$ (diamonds), and $(0.4 \lambda, 2.9 \lambda)$ (squares). (a) Experimental data as a function of the atom number. (b,c) Same data plotted as a function of the optical depth $b_{0}$ and of the central cloud density $\rho_{0}$. For all the measurements, the saturation parameter is $s=I / I_{\text {sat }} \simeq 27$. Error bars on the decay time (standard errors from the fit) are smaller than the marker size.

cooperativity, we acquire three different data sets, shown as different symbols in Fig. 2. They are taken in three different trapping geometries, leading to different cloud sizes (Appendix D). We plot the same data in Fig. 2(b) as a function of the optical depth along $x, b_{0}=\rho_{0} l_{x} \sqrt{2 \pi} \sigma_{\mathrm{sc}}$, and in Fig. 2(c) as a function of the peak density $\rho_{0}=$ $N /\left[(2 \pi)^{3 / 2} l_{x} l_{r}^{2}\right]\left(l_{x, r}\right.$ are Gaussian sizes). We observe that the data nearly collapse on a single curve when plotted as a function of atom number rather than as a function of optical depth. Furthermore, the decay times cannot be described by the cloud density only, which is a local quantity. This is expected given the long-range character of the dipoledipole interaction. We verify that the amplitude of the subradiant decay measured as the relative area in the tail (see Appendix F) is also governed by the atom number only. Therefore, the two parameters describing subradiance, namely, lifetime and amplitude, are solely governed by $N$, which is the cooperativity parameter for this regime, as explained in the Introduction. This behavior distinguishes our results from the ones in dilute systems, where the cooperativity parameter is $b_{0}$ [27], and indicates that we are approaching the Dicke limit. The imperfect collapse of the experimental data in Fig. 2(a) might be due to the fact that the system size along $x$ is still larger than the excitation wavelength.

\section{STUDY OF MULTIPLY EXCITED SUBRADIANT STATES}

A natural application of subradiance would be to store light in an atomic medium. Storing multiphoton states would require long-lived multiply excited states. Therefore, understanding the nature of these excitations is a prerequisite for the application of multiple excitation storage [12]. Here, we investigate this question experimentally by varying the intensity of the excitation laser.

Considering the strong driving limit first, the ensemble is prepared in a product state where each atom is in a mixture of the ground $|g\rangle$ and excited $|e\rangle$ states, with density matrix $\rho=\left(1 / 2^{N}\right)(|e\rangle\langle e|+| g\rangle\langle g|)^{\otimes N}$. In general, this mixture contains subradiant components, as was discussed in Ref. [12] for the case of two atoms. Reaching such a steady state during the excitation therefore leads to the initial excitation of long-lived subradiant states. However, it does not preclude the further population of these states during the early decay after switching off the laser excitation $[12,29,38]$. For large $N$, since we are dealing with two-level systems and not with classical dipoles, the fact that a single atom cannot be excited twice leads to nontrivial properties of multiply excited states. It was shown $[19,28-30]$ in the case of ordered 1D arrays that subradiant states containing $n_{\mathrm{exc}}>1$ excitations are built from a superposition of subradiant states of the single excitation manifold, which decay independently with their respective lifetime $\Gamma_{n}^{(1)}$, as exemplified for $n_{\text {exc }}=2$ in the caption of Fig. 3. The signal resulting from the decay of an $n_{\text {exc }}>1$ state is $\propto \sum_{n=1}^{n_{\text {exc }}} \exp \left(-\Gamma_{n}^{(1)} t\right)$. The interest of this ansatz stems from the fact that the singly excited states can be calculated via a model of classical coupled dipoles [39]. However, it is an open question whether this simple picture holds in the disordered case studied here. As we show 


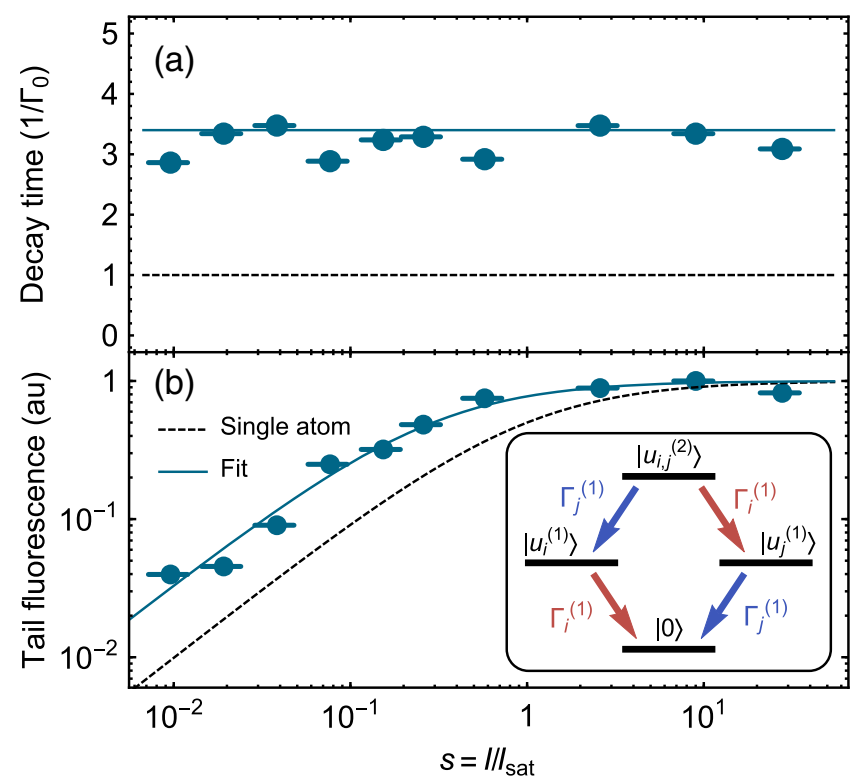

FIG. 3. (a) Measurement of the decay time of the subradiant tail (unit of $\Gamma_{0}^{-1}$ ) as a function of the saturation parameter $s$ of the excitation laser. Black dashed line: single-atom decay time. (b) Total number of counts recorded in the subradiant tail (normalized to the maximum value) as a function of $s$, together with a fit by a function $\alpha s /(1+\alpha s)$ (blue solid line). From the fit, we extract a decay time $\tau=\alpha \Gamma_{0}^{-1}$ (see text), which is shown as a solid blue line in panel (a). Black dashed line: single-atom response obtained by setting $\alpha=1$ in the previous equation. Inset: independent decay process of multiply excited subradiant states (here, exemplified with $n_{\text {exc }}=2$ ). Singly excited states decay independently at a rate given by decay rates of the singleexcitation subradiant eigenmodes.

below, our experimental findings support the picture of multiply excited subradiant states constructed as a superposition of independent, singly excited, subradiant states.

To control the number of excitations in the system, we vary the saturation parameter $s=I / I_{\text {sat }}$ between $s \simeq 0.01$ and $s \simeq 30$. For these measurements, we work without compression of the trap, and the atom number is fixed at about 4500. All the time traces acquired in this section are reported in Appendix H. We extract the subradiant lifetime using the procedure introduced earlier. In addition, we calculate the tail fluorescence by summing the signal for times larger than $t_{0}+\left(4 / \Gamma_{0}\right)$, where $t_{0}$ marks when the excitation pulse was switched off. We observe that the subradiant decay time is constant over 3 orders of magnitude of the excitation intensity [Fig. 3(a)] and that the tail fluorescence increases with excitation intensity before saturating at an intensity smaller than $I_{\text {sat }}$ [Fig. 3(b)]. The constant lifetime suggests a first simple description of the data as the excitation of a single mode. We thus use a single-mode approximation to describe the subradiant tail population with the following expression, assuming a saturation behavior similar to that of a single atom: $\alpha \alpha s /(1+\alpha s)$, with $\alpha$ the fit parameter. We report the result of this fit as the solid line in Fig. 3(b). From the extracted $\alpha=3.4(5)$ and assuming that the lifetime of a given mode $\tau^{\prime}$ dictates its saturation intensity $I_{\text {sat }}^{\prime} \propto 1 / \tau^{\prime}$ as for a single atom [40], we obtain $\tau^{\prime}=3.4 / \Gamma_{0}$, represented as a solid line in Fig. 3(a). This result agrees remarkably well with the direct measurement of the decay rate. Thus, the single-mode approximation describes our data very well, seeming to confirm the validity of this approximation. However, in the saturated regime, the long-lived modes leading to the subradiant decay host up to $10 \%$ of the total excitations (see Appendix F, Fig. 9), which for a fully saturated (i.e., $1 / 2$ excitation per atom) cloud of 5000 atoms, means several hundreds of excitations. Despite this large number of excitations, the decay rate remains the same, demonstrating that, in the subradiant tail, the rate at which excitations decay is independent of the density of excitations in the system. This finding is consistent with multiply excited states constructed from a large population of singly excited subradiant states that decay independently. In the opposite case of strong interactions between singly excited subradiant states, we would observe an excitation density-dependent decay rate due to additional decay processes induced by interactions between excitations. Experimentally, we observe average decay times of about $3 / \Gamma_{0}$, in agreement with the result of classical coupled-dipole calculations, showing that the single excitation manifold contains a large population of modes in the range around $3 / \Gamma_{0}$ [see Sec. V, Fig. 4(b)].

Finally, we come back to the description of the dynamics in terms of the mean-field model introduced in Sec. III. This model assumes a factorizable density matrix throughout the decay, and the coupling between atoms is induced by their dipole moment, which is proportional to the coherence between ground and excited states, $\rho_{\mathrm{eg}}$ [40]. However, for high saturation, the atoms are prepared in an incoherent mixture of the ground and excited states, hence, the coherence and the dipole vanish. Therefore, the meanfield model predicts a decoupling of the atoms, which then decay independently with the single-atom lifetime $1 / \Gamma_{0}$. Our observations up to $s=250$ (see Appendix G) contradict this prediction, showing that the density matrix of the system cannot be factorized throughout the decay, although it can be factorized initially.

\section{RELEASE OF THE SUBRADIANT EXCITATION}

In this final section, inspired by theoretical proposals [17-19], we perform a proof-of-principle demonstration of the on-demand release of the light stored in subradiant excitations. To do so, we apply a position-dependent detuning. The resulting inhomogeneous broadening makes the interaction between atoms no longer resonant: The ensemble now consists of independent atoms efficiently radiating, thus releasing the subradiant excitation.

To develop an intuition of how this protocol works, we first consider a toy model consisting of two coupled linear 
classical dipoles $d_{1}$ and $d_{2}$ with decay rate $\Gamma_{0}$ and separated by $\boldsymbol{r}_{12}$. The dynamics of the system is governed by the following equations (here, $\hbar=1$ ):

$$
\left(\begin{array}{l}
\dot{d}_{1} \\
\dot{d}_{2}
\end{array}\right)=\left(\begin{array}{cc}
-\frac{\Gamma_{0}}{2} & i V\left(\boldsymbol{r}_{12}\right) \\
i V\left(\boldsymbol{r}_{12}\right) & i \delta-\frac{\Gamma_{0}}{2}
\end{array}\right)\left(\begin{array}{l}
d_{1} \\
d_{2}
\end{array}\right),
$$

where $\delta$ is the difference between the transition frequencies of the two atoms and $V\left(\boldsymbol{r}_{12}\right)$ is the (complex) dipole-dipole interaction potential [26]. This system has super- and subradiant eigenmodes with decay rates $\Gamma_{ \pm}=\Gamma_{0} \pm$ $2 \operatorname{Im}\left[V\left(\boldsymbol{r}_{12}\right)\right]$. For $\delta=0$, these modes are the symmetric and antisymmetric combinations $\boldsymbol{v}_{ \pm} \propto(1, \pm 1)$. Hence, if at time $t=0$ the dipoles are prepared in $\boldsymbol{v}_{-}$, the turning on of the inhomogeneous broadening $(\delta \neq 0)$ projects $\boldsymbol{v}_{-}$onto the new basis provided by the eigenvectors of the matrix in Eq. (1). In the limit $\delta \gg\left|V\left(r_{12}\right)\right|$, these are the uncoupled dipoles $\boldsymbol{v}_{1}=$ $(1,0)$ and $\boldsymbol{v}_{2}=(0,1)$, with identical decay rate $\Gamma_{0}$. The evolution of the two-atom dipole is then given by $\boldsymbol{v}(t) \propto e^{-\Gamma_{0} t / 2}\left(\boldsymbol{v}_{1}-e^{i \delta t} \boldsymbol{v}_{2}\right)$, recovering the single-atom decay rate of the radiated power $v^{2}(t) \propto e^{-\Gamma_{0} t}$. This toy model thus shows that placing the atoms out of resonance with one another allows the system to radiate again as an assembly of independent emitters.

In Fig. 4(a), we report the results of the experiment obtained by applying an inhomogeneous broadening. To do so, we turn on the trap light at different times (indicated by the vertical arrows) after the extinction of the excitation laser. The black curve is our reference for which the trap is turned on at long times $(\simeq 1 \mu \mathrm{s})$. The far off-resonant light induces a position-dependent detuning described by

$\delta_{i}\left(x_{i}, y_{i}, z_{i}\right)=\frac{\delta_{0}}{1+x_{i}^{2} / x_{R}^{2}} \exp \left[-\frac{2\left(y_{i}^{2}+z_{i}^{2}\right)}{w_{\text {trap }}^{2}\left(1+x_{i}^{2} / x_{R}^{2}\right)}\right]$,

where $\delta_{0}$ is the light shift induced by the trap. Experimentally, $\delta_{0} \simeq 32 \Gamma_{0}, \quad w_{\text {trap }}=2.5 \mu \mathrm{m}, \quad$ and $x_{R}=$ $\pi w_{\text {trap }}^{2} / \lambda_{\text {trap }}$. Using the atomic density distribution in Eq. (2) for $x_{i}, y_{i}, z_{i}$, the standard deviation of the detuning induced by the trap is about $4 \Gamma_{0}$. After turning on this inhomogeneous broadening, we observe the emission of a pulse of light [Fig. 4(a)]. The presence of this pulse can be qualitatively understood using the toy model: When the inhomogeneous broadening is applied, the atoms start to radiate at a rate faster than the subradiant one; thus, the intensity of the emitted light is initially enhanced before decaying. Moreover, we observe that the pulse is followed by a faster decay, similar to the single-atom regime [fitting the experimental data after this pulse, we obtain a decay rate of 1.3(1) $\Gamma_{0}$ for all data sets]. The measurements have been performed with $\simeq 5000$ atoms and with $s \simeq 27$. We verified the collective origin of this effect by performing the same measurements for low atom number, observing no pulse. Furthermore, the strong suppression of subradiance
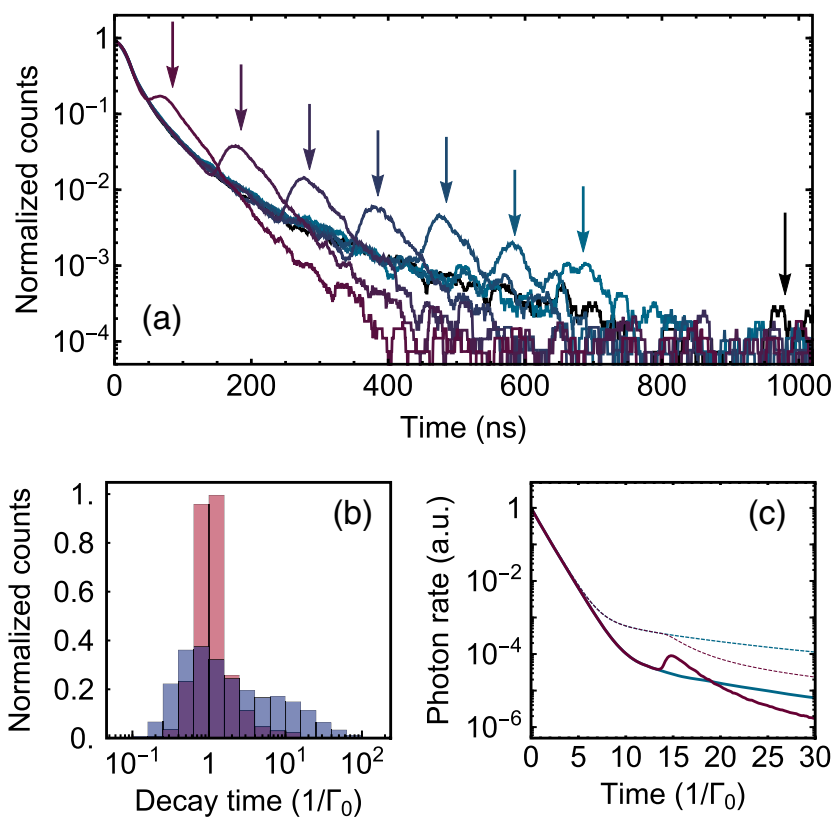

FIG. 4. Release of the light stored in subradiant excitations. (a) Experimental realization, each time trace represents an experimental sequence where the inhomogeneous broadening is applied at a different time, highlighted by the respective arrows. (b) Histogram of the decay times of the collective modes evaluated from the eigenvalues of the interaction matrix for 5000 atoms with the same density distribution as in the experiment, for ten realizations. Red (blue) histograms are calculated with (without) inhomogeneous broadening. (c) Results of the mean-field nonlinear coupled dipole simulations. Dashed lines: temporal evolution of the total excited state population $p(t)$. Solid lines: intensity $-d p / d t$ emitted in a $4 \pi$ solid angle. Purple lines: case where the inhomogeneous broadening is applied at $t=12.5 / \Gamma_{0}$. Blue lines: no inhomogeneous broadening applied.

obtained with a light shift varying slowly in space demonstrates that the subradiant excitations are not stored in pairs of close-by atoms but rather delocalized over all the atoms of the cloud [41]. This is expected for a near-resonant excitation of the cloud, as delocalized excitations correspond to states with small interaction frequency shifts.

To go deeper into the understanding of the observed behavior, we extend the toy model previously introduced to large ensembles. We obtain the decay rates by evaluating the eigenvalues $\lambda_{i}$ of the interaction matrix for $N=5000$ classical coupled dipoles sampled using the experimental position-dependent detuning. The associated modes are single-excitation modes, but as observed earlier, they should give a qualitative description of the behavior of the subradiant tail. The results are shown in Fig. 4(b). They indicate that, in the presence of the inhomogeneous broadening, the distribution of decay times is much narrower than in free space [note the log scale in Fig. 4(b)]. In particular, a significant fraction of modes with decay rates close to the observed subradiant one (between $\Gamma_{0} / 3$ and $\left.\Gamma_{0} / 10\right)$ are suppressed by the inhomogeneous broadening. 


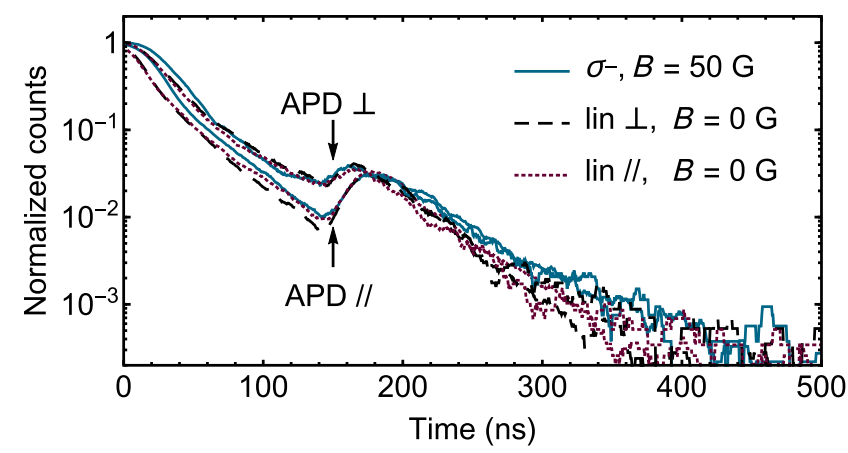

FIG. 5. Directionality of the emitted light pulse. Release of the subradiant excitation, observed with light collected axially $(\mathrm{APD} / /)$ (bottom curves) and radially (APD $\perp$ ) (top curves). The experiment is performed with a linear polarization of the excitation light, either parallel (dotted lines) or perpendicular (dashed lines) to the cloud, and with a circular polarization in the presence of a 50-G magnetic field aligned along the excitation direction (solid lines).

We further perform numerical simulations of the dynamics for smaller atom numbers using the mean-field model already introduced in Sec. III. The results are shown in Fig. 4(c) for a cloud with $N=200$ atoms at a density $\rho_{0} / k^{3}=0.3$. The trap is turned on during the decay. We take into account the finite rise time of the trap beam of about $25 \mathrm{~ns}$. The simulation provides the evolution of the total population of the excited state $p(t)=\sum_{i=1}^{N} \rho_{\mathrm{ee}}^{(i)}(t)$ (dashed line). However, experimentally, we measure the intensity of the light emitted by the cloud, which, in the absence of a drive, is proportional to $-d p(t) / d t$ (in a $4 \pi$ solid angle), represented by the continuous lines of Fig. 4(c). When the inhomogeneous broadening is applied, the population curve presents a change of slope, corresponding to a peak in the intensity, followed by a decay at a rate that is now close to the single-atom one. These simulations confirm the interpretation of our experimental findings.

Finally, as shown in Fig. 5, we observe that the enhancement of the emission is stronger along the cloud axis. We have further observed, by changing polarization and the internal structure of the atoms, that no significant difference arises in the released pulse shape, Fig. 5. We leave for future work the investigation of how to control the directionality of the pulse, which could allow for the tailoring of the angular emission pattern of the excitation stored in subradiance.

\section{CONCLUSION}

In this work, we investigated the subradiant decay of excitations stored in a dense cloud of atoms trapped in an optical tweezer, approaching the Dicke limit of a large number of atoms in a volume smaller than $\lambda^{3}$. We confirm that the cooperativity parameter is the atom number rather than the optical depth or the density. Moreover, by tuning the intensity of the excitation laser, we studied the nature of multiply excited subradiant states and experimentally found that all the information is contained in the response of the system at low intensity, where only singly excited modes are populated. A quantitative theoretical description in our regime is, however, extremely challenging, and new models describing this interacting dissipative many-body system need to be developed. Finally, by applying an inhomogeneous broadening, we were able to release the subradiant excitation stored in the cloud in the form of a pulse of light. Our experiment thus represents a proof of principle of the temporal control of subradiance in an atomic medium, a prerequisite for its use as a light storage medium. It was made possible by the small size of the cloud, which permitted the use of relatively low power of the control light to apply a significant detuning between atoms. In the future, it could be applied to systems featuring tailored subradiant modes, such as structured atomic systems [19,42-44], in which these modes could be selectively excited by a local addressing.

\section{ACKNOWLEDGMENTS}

We thank Ana Asenjo-Garcia, Stuart Masson, Luis Orozco, Robin Kaiser, and William Guerin for discussions. This project has received funding from the European Union's Horizon 2020 research and innovation program under Grant Agreement No. 817482 (PASQuanS) and by the Région Îlede-France in the framework of DIM SIRTEQ (project DSHAPE). A. G. is supported by the Délégation Générale de l'Armement Fellowship No. 2018.60.0027.

\section{APPENDIX A: NONLINEAR COUPLED-DIPOLE MODEL}

In this section, we provide some details about the meanfield, nonlinear, coupled-dipole model used in the main text. A derivation of the main equations can be found elsewhere [31,34]. We consider an ensemble of $N$ two-level atoms at position $\boldsymbol{r}_{n}$ coupled to light by a transition with dipole element $d_{0}$, detuning $\Delta$, and natural broadening $\Gamma_{0}$. Assuming that the density matrix can be factorized, $\rho=\bigotimes_{n} \rho_{n}$, the dynamics is governed by a system of coupled OBEs:

$$
\begin{array}{r}
\frac{d \rho_{\mathrm{ee}, \mathrm{n}}}{d t}=-\Gamma_{0} \rho_{\mathrm{ee}, n}+i \rho_{\mathrm{ge}, n} \frac{\Omega_{n}}{2}-i \rho_{\mathrm{eg}, n} \frac{\Omega_{n}^{*}}{2}, \\
\frac{d \rho_{\mathrm{eg}, n}}{d t}=\left(i \Delta-\frac{\Gamma_{0}}{2}\right) \rho_{\mathrm{eg}, n}-i \Omega_{n}\left(\rho_{\mathrm{ee}, n}-\rho_{\mathrm{gg}, n}\right),
\end{array}
$$

where $\rho_{\alpha \beta, \mathrm{n}}$ represents the elements of the density matrix of the $n$th atom. The driving Rabi frequency $\Omega_{n}$ experienced by atom $n$ is the sum of the laser driving and the field scattered by other dipoles: 


$$
\begin{aligned}
\Omega_{n} & =\Omega_{\text {laser }}\left(\boldsymbol{r}_{n}\right)+i \sum_{m \neq n} \rho_{\mathrm{eg}, m} V\left(\boldsymbol{r}_{n}-\boldsymbol{r}_{m}\right), \\
V(\boldsymbol{r}) & =\frac{3}{2 i} \frac{e^{i k r}}{k r}\left[p(\hat{r})+q(\hat{r})\left(\frac{i}{k r}-\frac{1}{(k r)^{2}}\right)\right],
\end{aligned}
$$

where $p(\hat{r})$ and $q(\hat{r})$ depend on the polarization of the driving field [25].

In the strong driving limit, $\rho_{\mathrm{eg}, n}$ vanishes. Moreover, when the driving is turned off $\left(\Omega_{\text {laser }}=0\right)$, at any subsequent time, $\rho_{\mathrm{eg}, n}=0$, and the equations are decoupled, leading to the single-atom decay.

\section{APPENDIX B: DEPENDENCE OF SUBRADIANCE ON DETUNING}

All the measurements reported in the main text have been performed on resonance. To demonstrate that our observations cannot be explained by radiation trapping, which depends strongly on detuning [11], we have also acquired three different data sets at three different detunings of the driving laser. In Fig. 6, we report these measurements performed at resonance $\Delta / \Gamma_{0}=0, \Delta / \Gamma_{0}=1$, and $\Delta / \Gamma_{0}=3$. At $\Delta / \Gamma_{0}=3$, we observe a small reduction in fluorescence at large detunings even at this intensity. However, the tail of each distribution behaves very similarly, independently of the detuning.

\section{APPENDIX C: FITTING PROCEDURE TO EXTRACT THE SUBRADIANT LIFETIME}

In this section, we provide more details about the fitting procedure used to analyze the experimental data. In Fig. 7(a), we report an example of a fluorescence trace acquired in the small-atom-number regime together with a fit that makes use of a single exponential decay. We fit all the data in this way, and for every measurement, we evaluate the reduced $\chi^{2}$ :

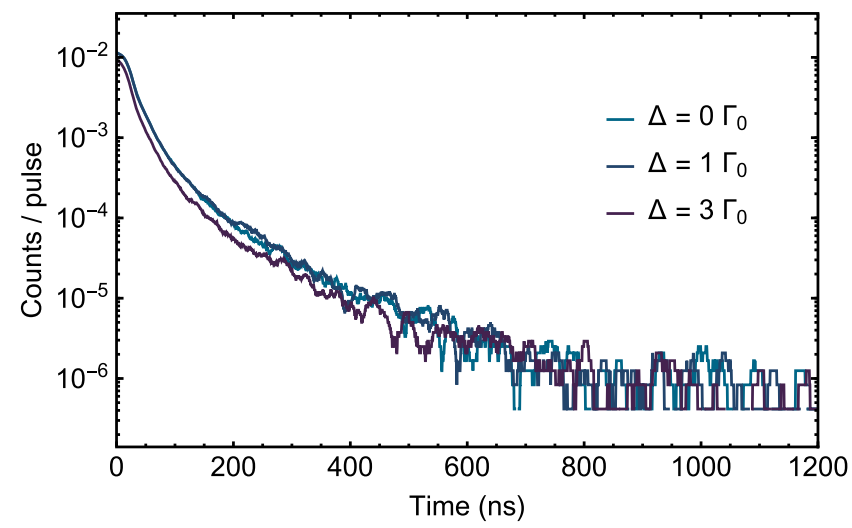

FIG. 6. Photon-count traces acquired as a function of the excitation frequency. The experimental data have been acquired in the shallowest trapping geometry described in the main text with $N \simeq 4500$. The temporal traces have been divided by the number of pulses of light used; thus, they represent the mean number of photons collected during one pulse of resonant light.

$$
\chi^{2}=\frac{1}{K_{\text {bin }}} \sum_{i=1}^{K_{\text {bin }}} \frac{\left(N_{i}^{\text {exp }}-N^{\text {the }}\left(t_{i}\right)\right)^{2}}{N^{\text {the }}\left(t_{i}\right)}
$$

where $K_{\text {bin }}$ is the number of time bins in the data set (500 ps bins), $N_{i}^{\exp }$ is the recorded number of counts in the bin centered on time $t_{i}$, and $N^{\text {the }}\left(t_{i}\right)$ is the value of the fitting function. This definition of $\chi^{2}$ assumes Poissonian statistics for the recorded counts in each bin.

In Fig. 7(c), we report the values of $\chi^{2}$ as a function of the atom number, for the shallowest trapping geometry described in the main text (blue points in Fig. 2). As the atom number in the cloud becomes larger, the system exhibits super- and subradiance, and the fit with a single exponential decay is no longer able to describe the entire observed dynamics as revealed by an increase in $\chi^{2}$. When
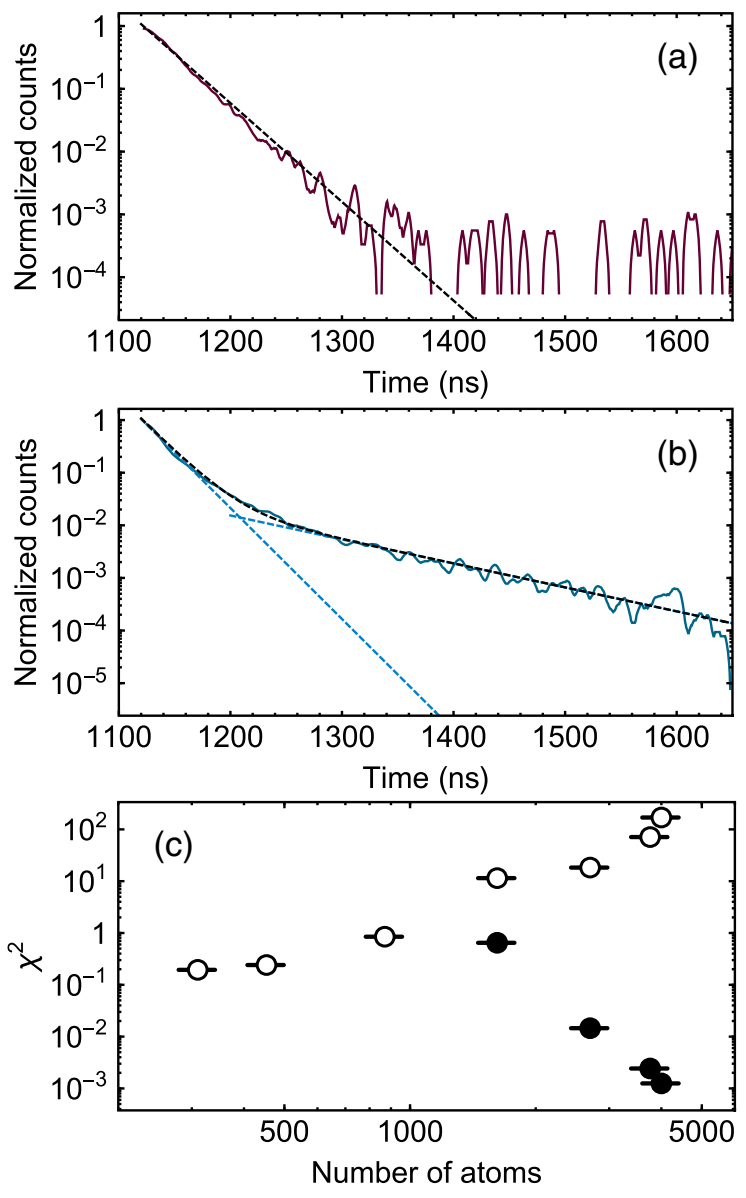

FIG. 7. (a) Solid line: example of decay in the small-atomnumber regime $(N \simeq 300)$ shown with the fit using a single exponential decay (dashed line). (b) Solid line: decay in the largeatom-number regime $(N \simeq 4000)$, dashed line: phenomenological fit with the sum of two exponential decays. The dotted lines represent the two different decay rates, i.e., super- and subradiance. The traces in panels (a) and (b) have been normalized to the steady state. (c) Values of $\chi^{2}$ obtained with the doubleexponential (filled circles) or a single-exponential decay (empty markers) versus atom number. 
it becomes larger than 1, we use a different phenomenological model to describe the decay, fitting with the sum of two exponential decays [see Fig. 7(b)]. The agreement between this second model and the experimental data is much better in the large-atom-number regime, and consequently, $\chi^{2}$ is smaller, as highlighted by the filled points in Fig. $7(\mathrm{c})$. The very small values of $\chi^{2}$ come from the large uncertainties resulting from the small number of counts in the tail.

\section{APPENDIX D: THE THREE DIFFERENT TRAPPING GEOMETRIES}

In Sec. III, we employ three different trapping geometries that allow us to obtain three different cloud dimensions. In the first one, the trap is used to load the atoms from the MOT (Gaussian sizes $l_{r} \simeq 0.7 \lambda, l_{x} \simeq 7.7 \lambda$, blue circles). In the second one, we increase the power of the trapping beam by about $60 \%$, gaining a factor 2 in density without losing atoms $\left(l_{r} \simeq 0.5 \lambda, l_{x} \simeq 6.0 \lambda\right.$, purple diamonds). In the third case, we reduce the beam waist down to $1.8 \mu \mathrm{m}$. The atom number after the compression is lower $(N \simeq 1500)$, but we reach a high density, $\rho_{0} / k^{3} \simeq 1.5$, owing to the reduced trapping volume $\left(l_{r} \simeq 0.4 \lambda, \quad l_{x} \simeq 2.9 \lambda\right.$, black squares) [45]. A complete set of time traces can be found below. For every point, we measure the atom number and the temperature.

\section{APPENDIX E: EFFECT OF POLARIZATION AND OPTICAL PUMPING}

Unless otherwise specified, the measurements reported in this work are performed with a linearly polarized excitation light without any direct optical pumping (OP). We did not observe that the polarization direction impacts the observed subradiance. However, there could be an

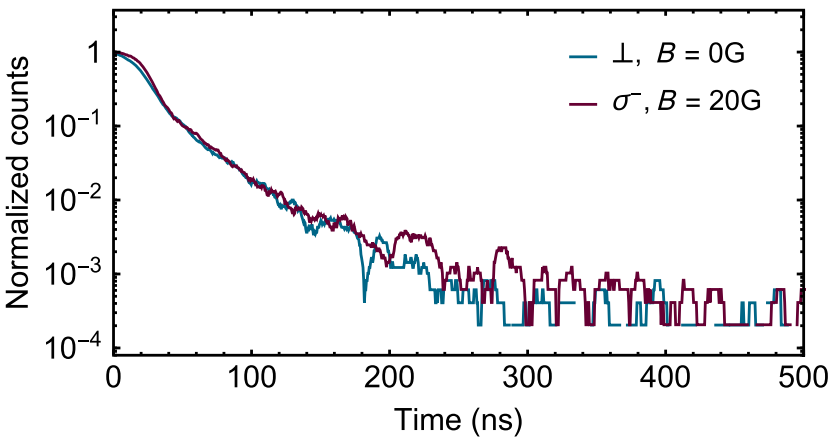

FIG. 8. Photon-count decay with a linear polarization, no magnetic field, and no optical pumping, i.e., multilevel situation (blue). The same is shown for the two-level case: 20-G magnetic field, $\sigma^{-}$polarization, and prior optical pumping (purple). The two traces have been normalized to the steady state, with measurements for $N \simeq 4500$ in the first trapping geometry (see Appendix D). effect due to the fact that the linear polarization configuration does not correspond to a two-level configuration.

It was recently shown [46] that on a $J=0 \rightarrow J=1$ transition, subradiance can be modified at high density with respect to the two-level scalar model predictions. In our case, in order to check if the level structure strongly modifies the collective behavior hosted in the tail of the fluorescence signal, we perform one experiment in a situation much closer to a two-level configuration: a circularly polarized $\left(\sigma^{-}\right)$excitation, together with a $20-\mathrm{G}$ magnetic field along the excitation propagation axis. We perform hyperfine and Zeeman optical pumping with the same polarization as the excitation light to place ourselves in a closed two-level system (between $F=2, m_{F}=-2$, $F^{\prime}=3$, and $m_{F}=-3$ ). The light scattered by an atom onto a nearby atom might contain other polarization components and drive other transitions out of the two-level system. However, the detuning between the $\sigma^{-}$and $\pi$ polarization transitions (closest in detuning) is about $5 \Gamma_{0}$, preventing the rescattering of this polarization by nearby atoms.
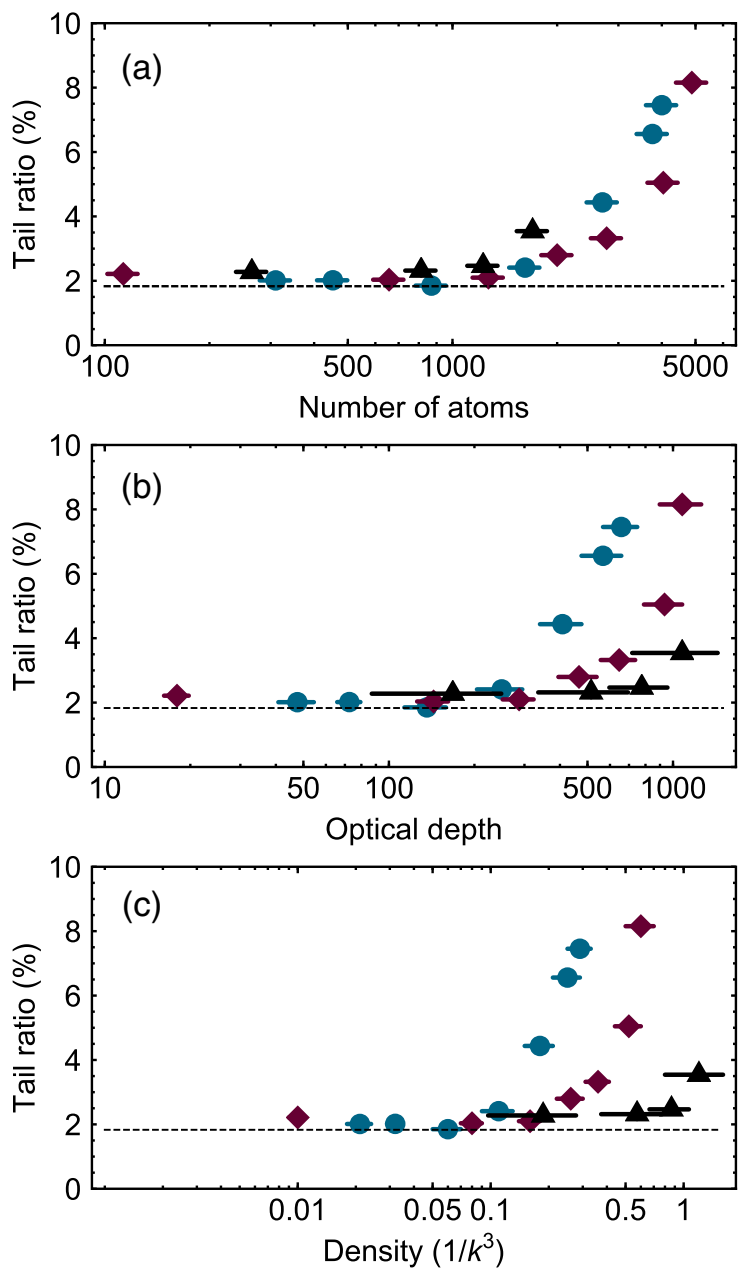

FIG. 9. Tail ratio as defined by Eq. (F) for the same data sets as used to extract the decay times in Fig. $2(s \simeq 27)$. 
In Fig. 8, we show in blue an acquisition from linear polarization and without optical pumping, and in purple that with optical pumping, circular polarization, and magnetic field. We observe that the tails of the two traces are similar, within our dynamic range of observation. We conclude that the internal structure does not seem to strongly affect the results on subradiance reported in this work or our conclusions.

\section{APPENDIX F: RELATIVE POPULATION OF THE LONG-LIVED STATES}

Another parameter that we use to quantify subradiance is the relative fluorescence observed in the long-lived tail with respect to the total fluorescence recorded after switching off the drive. We refer to this quantity as the tail ratio. It is defined as TR $=\left[\int_{\frac{4}{\Gamma_{0}}}^{\infty} I(t) d t / \int_{0}^{\infty} I(t) d t\right]$, where $I(t)$ represents the time-resolved fluorescence emitted by the atomic cloud, with $t=0$ the switch-off time of the excitation laser. This parameter estimates the fraction of excitation still hosted in the system $4 / \Gamma_{0} \simeq 100 \mathrm{~ns}$ after switching off the excitation, compared to the whole decay. In the single-atom limit, this parameter is equal to $e^{-4} \simeq 2 \%$ by definition.

We analyze the behavior of this tail ratio as a function of atom number on the same data as used for Fig. 2. In Fig. 9, we indeed see the collapse as a function of atom number, similarly to what was found for the decay time (see discussion in Sec. III).

\section{APPENDIX G: MEASUREMENTS AT LARGE SATURATION INTENSITY}

In this section, we report the data acquired at very high values of the drive intensity, reaching $s=I / I_{\text {sat }} \simeq 250$. The measurements have been performed with a larger atom number, $N \simeq 6000$. For this reason, this data set and the one used for Fig. 3 of the main text cannot be directly compared. In Fig. 10(a), we report the temporal traces acquired in this way, normalized to the steady-state value of the trace taken with the largest excitation intensity. In
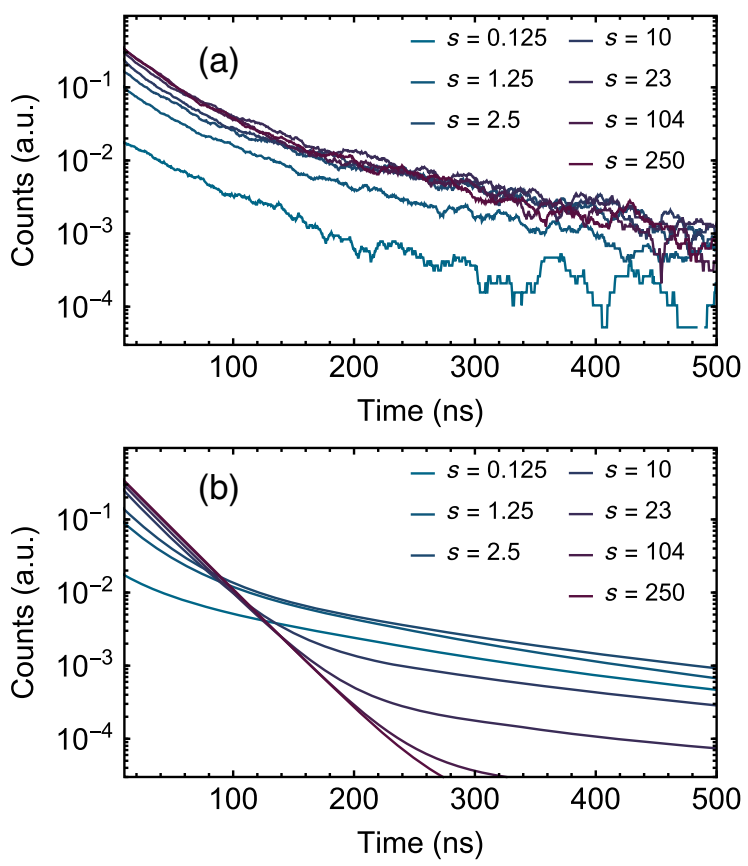

FIG. 10. (a) Photon-count decays acquired for different excitation intensities. (b) Numerical simulations performed with a nonlinear coupled-dipole model, using 100 atoms at a density $\rho_{0} / k^{3}=0.3$. The traces have been normalized to the steady-state value of the measurement at largest intensity. Here, we define the origin of the time $(t=0) 30 \mathrm{~ns}$ after switching off the excitation.

Fig. 10(b), we instead report the results of numerical simulations performed using the nonlinear coupled-dipole model, with 100 atoms distributed in a Gaussian cloud with a peak density $\rho_{0}=0.3 / k^{3}$. The reported lines are the results of ten realizations of the same numerical experiment. As one can see, according to the mean-field model, the subradiance is expected to disappear as the excitation strength is increased. The fact that, experimentally, the system still hosts a subradiant excitation even at very large intensity shows that the density matrix of the system cannot be factorized throughout the decay.
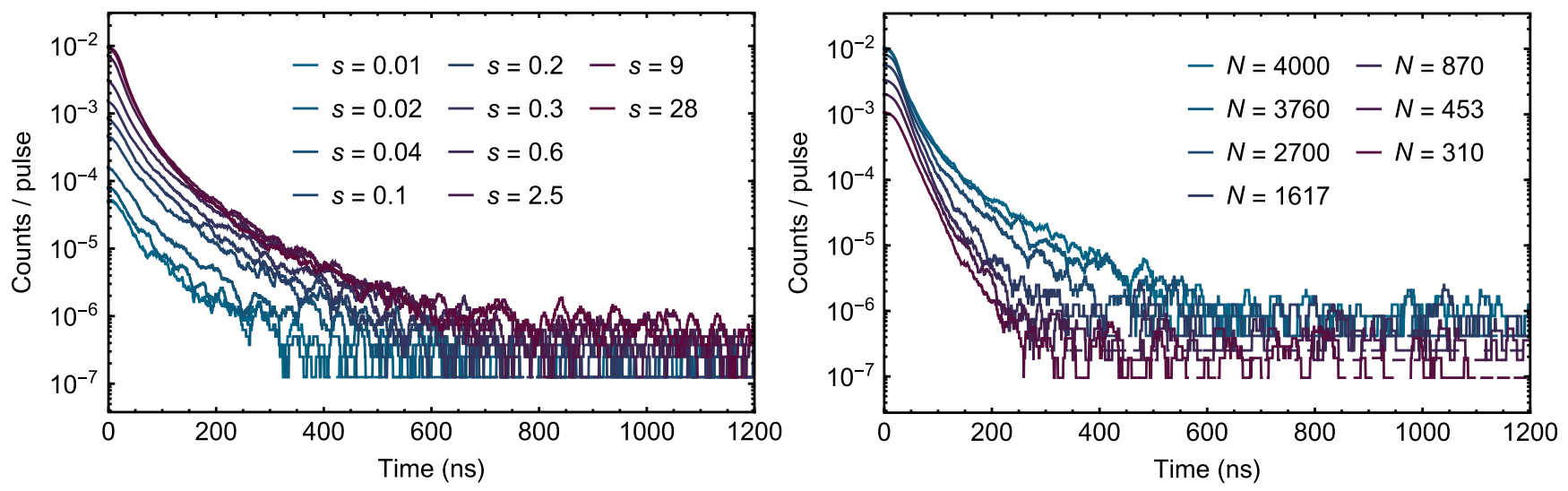

FIG. 11. Left panel: photon-count decays acquired for different drive intensities. Right panel: photon-count decays acquired as a function of the atom number for the first trapping geometry (circles in Figs. 2 and 9). 


\section{APPENDIX H: FLUORESCENCE DECAY CURVES}

For completeness, in Fig. 11, we report the experimental photon-count decay curves used in the analysis described in the main text. In the left panel, we show the ones measured as a function of atom number, and in the right panel, for different drive intensities.

[1] R. H. Dicke, Coherence in Spontaneous Radiation Processes, Phys. Rev. 93, 99 (1954).

[2] M. Gross and S. Haroche, Superradiance: An Essay on the Theory of Collective Spontaneous Emission, Phys. Rep. 93, 301 (1982).

[3] R. G. DeVoe and R. G. Brewer, Observation of Superradiant and Subradiant Spontaneous Emission of Two Trapped Ions, Phys. Rev. Lett. 76, 2049 (1996).

[4] M. O. Araújo, I. Krešić, R. Kaiser, and W. Guerin, Superradiance in a Large and Dilute Cloud of Cold Atoms in the Linear-Optics Regime, Phys. Rev. Lett. 117, 073002 (2016).

[5] S. J. Roof, K. J. Kemp, M. D. Havey, and I. M. Sokolov, Observation of Single-Photon Superradiance and the Cooperative Lamb Shift in an Extended Sample of Cold Atoms, Phys. Rev. Lett. 117, 073003 (2016).

[6] P. Solano, P. Barberis-Blostein, F. K. Fatemi, L. A. Orozco, and S. L. Rolston, Super-Radiance Reveals Infinite-Range Dipole Interactions through a Nanofiber, Nat. Commun. 8, 1857 (2017).

[7] D. Pavolini, A. Crubellier, P. Pillet, L. Cabaret, and S. Liberman, Experimental Evidence for Subradiance, Phys. Rev. Lett. 54, 1917 (1985).

[8] C. Hettich, C. Schmitt, J. Zitzmann, S. Kühn, I. Gerhardt, and V. Sandoghdar, Nanometer Resolution and Coherent Optical Dipole Coupling of Two Individual Molecules, Science 298, 385 (2002).

[9] Y. Takasu, Y. Saito, Y. Takahashi, M. Borkowski, R. Ciuryło, and P. S. Julienne, Controlled Production of Subradiant States of a Diatomic Molecule in an Optical Lattice, Phys. Rev. Lett. 108, 173002 (2012).

[10] B. H. McGuyer, M. McDonald, G. Z. Iwata, M. G. Tarallo, W. Skomorowski, R. Moszynski, and T. Zelevinsky, Precise Study of Asymptotic Physics with Subradiant Ultracold Molecules, Nat. Phys. 11, 32 (2015).

[11] W. Guerin, M. O. Araújo, and R. Kaiser, Subradiance in a Large Cloud of Cold Atoms, Phys. Rev. Lett. 116, 083601 (2016).

[12] A. Cipris, N. A. Moreira, T. S. do Espirito Santo, P. Weiss, C. J. Villas-Boas, R. Kaiser, W. Guerin, and R. Bachelard, Subradiance with Saturated Atoms: Population Enhancement of the Long-Lived States, Phys. Rev. Lett. 126, 103604 (2021).

[13] D. Das, B. Lemberger, and D. D. Yavuz, Subradiance and Superradiance-to-Subradiance Transition in Dilute Atomic Clouds, Phys. Rev. A 102, 043708 (2020).

[14] N. Stiesdal, H. Busche, J. Kumlin, K. Kleinbeck, H. P. Büchler, and S. Hofferberth, Observation of Collective Decay Dynamics of a Single Rydberg Superatom, Phys. Rev. Research 2, 043339 (2020).
[15] J. Rui, D. Wei, A. Rubio-Abadal, S. Hollerith, J. Zeiher, D. M. Stamper-Kurn, C. Gross, and I. Bloch, A Subradiant Optical Mirror Formed by a Single Structured Atomic Layer, Nature (London) 583, 369 (2020).

[16] D. Plankensteiner, L. Ostermann, H. Ritsch, and C. Genes, Selective Protected State Preparation of Coupled Dissipative Quantum Emitters, Sci. Rep. 5, 16231 (2015).

[17] G. Facchinetti, S. D. Jenkins, and J. Ruostekoski, Storing Light with Subradiant Correlations in Arrays of Atoms, Phys. Rev. Lett. 117, 243601 (2016).

[18] H. H. Jen, M.-S. Chang, and Y.-C. Chen, Cooperative Single-Photon Subradiant States, Phys. Rev. A 94, 013803 (2016).

[19] A. Asenjo-Garcia, M. Moreno-Cardoner, A. Albrecht, H. J. Kimble, and D. E. Chang, Exponential Improvement in Photon Storage Fidelities Using Subradiance and "Selective Radiance" in Atomic Arrays, Phys. Rev. X 7, 031024 (2017).

[20] J. A. Needham, I. Lesanovsky, and B. Olmos, SubradianceProtected Excitation Transport, New J. Phys. 21, 073061 (2019).

[21] L. Ostermann, H. Ritsch, and C. Genes, Protected State Enhanced Quantum Metrology with Interacting Two-Level Ensembles, Phys. Rev. Lett. 111, 123601 (2013).

[22] G. Facchinetti and J. Ruostekoski, Interaction of Light with Planar Lattices of Atoms: Reflection, Transmission, and Cooperative Magnetometry, Phys. Rev. A 97, 023833 (2018).

[23] D. S. Wild, E. Shahmoon, S. F. Yelin, and M. D. Lukin, Quantum Nonlinear Optics in Atomically Thin Materials, Phys. Rev. Lett. 121, 123606 (2018).

[24] P.-O. Guimond, A. Grankin, D. V. Vasilyev, B. Vermersch, and P. Zoller, Subradiant Bell States in Distant Atomic Arrays, Phys. Rev. Lett. 122, 093601 (2019).

[25] E. Akkermans, A. Gero, and R. Kaiser, Photon Localization and Dicke Superradiance in Atomic Gases, Phys. Rev. Lett. 101, 103602 (2008).

[26] J. D. Jackson, Classical Electrodynamics (John Wiley \& Sons, New York, 2007).

[27] W. Guerin, M. Rouabah, and R. Kaiser, Light Interacting with Atomic Ensembles: Collective, Cooperative and Mesoscopic Effects, J. Mod. Opt. 64, 895 (2017).

[28] A. Albrecht, L. Henriet, A. Asenjo-Garcia, P. B. Dieterle, O. Painter, and D. E. Chang, Subradiant States of Quantum Bits Coupled to a One-Dimensional Waveguide, New J. Phys. 21, 025003 (2019).

[29] L. Henriet, J. S. Douglas, D. E. Chang, and A. Albrecht, Critical Open-System Dynamics in a One-Dimensional Optical-Lattice Clock, Phys. Rev. A 99, 023802 (2019).

[30] Y.-X. Zhang and K. Mølmer, Theory of Subradiant States of a One-Dimensional Two-Level Atom Chain, Phys. Rev. Lett. 122, 203605 (2019).

[31] A. Glicenstein, G. Ferioli, N. Šibalić, L. Brossard, I. FerrierBarbut, and A. Browaeys, Collective Shift in Resonant Light Scattering by a One-Dimensional Atomic Chain, Phys. Rev. Lett. 124, 253602 (2020).

[32] A. Glicenstein, G. Ferioli, L. Brossard, Y. R. P. Sortais, D. Barredo, F. Nogrette, I. Ferrier-Barbut, and A. Browaeys, Fast and Efficient Preparation of $1 D$ Chains and Dense Cold Atomic Clouds, arXiv:2101.07544. 
[33] N. Bruno, L. C. Bianchet, V. Prakash, N. Li, N. Alves, and M. W. Mitchell, Maltese Cross Coupling to Individual Cold Atoms in Free Space, Opt. Express 27, 31042 (2019).

[34] T. S. do Espirito Santo, P. Weiss, A. Cipris, R. Kaiser, W. Guerin, R. Bachelard, and J. Schachenmayer, Collective Excitation Dynamics of a Cold Atom Cloud, Phys. Rev. A 101, 013617 (2020).

[35] G. Labeyrie, E. Vaujour, C. A. Müller, D. Delande, C. Miniatura, D. Wilkowski, and R. Kaiser, Slow Diffusion of Light in a Cold Atomic Cloud, Phys. Rev. Lett. 91, 223904 (2003).

[36] S. Krämer and H. Ritsch, Generalized Mean-Field Approach to Simulate the Dynamics of Large Open Spin Ensembles with Long Range Interactions, Eur. Phys. J. D 69, 282 (2015).

[37] C. D. Parmee and N. R. Cooper, Phases of Driven TwoLevel Systems with Nonlocal Dissipation, Phys. Rev. A 97, 053616 (2018).

[38] S. J. Masson, I. Ferrier-Barbut, L. A. Orozco, A. Browaeys, and A. Asenjo-Garcia, Many-Body Signatures of Collective Decay in Atomic Chains, Phys. Rev. Lett. 125, 263601 (2020).

[39] J. Ruostekoski and J. Javanainen, Quantum Field Theory of Cooperative Atom Response: Low Light Intensity, Phys. Rev. A 55, 513 (1997).
[40] G. Grynberg, A. Aspect, and C. Fabre, Introduction to Quantum Optics (Cambridge University Press, Cambridge, England, 2010).

[41] N. J. Schilder, C. Sauvan, J.-P. Hugonin, S. Jennewein, Y. R. P. Sortais, A. Browaeys, and J.-J. Greffet, Polaritonic Modes in a Dense Cloud of Cold Atoms, Phys. Rev. A 93, 063835 (2016).

[42] R. J. Bettles, S. A. Gardiner, and C. S. Adams, Cooperative Eigenmodes and Scattering in One-Dimensional Atomic Arrays, Phys. Rev. A 94, 043844 (2016).

[43] R. J. Bettles, S. A. Gardiner, and C. S. Adams, Enhanced Optical Cross Section via Collective Coupling of Atomic Dipoles in a 2D Array, Phys. Rev. Lett. 116, 103602 (2016).

[44] E. Shahmoon, D. S. Wild, M. D. Lukin, and S. F. Yelin, Cooperative Resonances in Light Scattering from TwoDimensional Atomic Arrays, Phys. Rev. Lett. 118, 113601 (2017).

[45] A. Glicenstein, G. Ferioli, L. Brossard, Y. R. P. Sortais, D. Barredo, F. Nogrette, I. Ferrier-Barbut, and A. Browaeys, Fast and Efficient Preparation of $1 D$ Chains and Dense Atomic Clouds, arXiv:2101.07544.

[46] A. Cipris, R. Bachelard, R. Kaiser, and W. Guerin, Van der Waals Dephasing for Dicke Subradiance in Cold Atomic Clouds, arXiv:2012.06248. 